\title{
Antiviral Therapy in Chronic Hepatitis B With Mild Acute Exacerbation
}

\author{
Su Lin ${ }^{\mathrm{a}}$, Qiaoxia Ye ${ }^{\mathrm{a}, \mathrm{b}}$, Mingfang Wang ${ }^{\mathrm{a}}$, Yinlian Wu $\mathrm{Wu}^{\mathrm{a}}$, Zhiyuan Weng ${ }^{\mathrm{c}}$, Yueyong Zhu ${ }^{\mathrm{a}, \mathrm{d}}$
}

\begin{abstract}
Background: The aim of this study was to assess the efficacy and safety of peginterferon $\alpha-2 \mathrm{a}($ pegIFN) and nucleos(t)ide analogues (NA) treatments in patients with hepatitis $\mathrm{B}$ envelope antigen (HBeAg)positive chronic hepatitis $\mathrm{B}(\mathrm{CHB})$ with mild acute exacerbation (AE).

Methods: Treatment-naive $\mathrm{HBeAg}$-positive $\mathrm{CHB}$ patients with $\mathrm{AE}$ who received pegIFN or NA (entecavir (ETV) or telbivudine (LDT)) therapies were retrospectively selected. The $\mathrm{HBeAg}$ seroconversion rate, hepatitis B surface antigen ( $\mathrm{HBsAg}$ ) loss rate and the cost-effectiveness of different treatments were compared.

Results: A total of 63 patients with pegIFN therapy and 78 with NA ( 38 with ETV and 40 with LDT) therapy were included. The HBsAg loss rate was significantly higher in the pegIFN group when compared with the NA group (on week 96: 9/63 (14.29\%) vs. 1/78 (1.28\%), P $=0.005)$. No significant difference in hepatitis B virus (HBV) DNA negativity or the $\mathrm{HBeAg} / \mathrm{HBsAg}$ seroconversion rate was found between ETV and LDT group. One year of pegIFN therapy resulted in 18.56 quality-adjusted life years (QALYs) per patient, and the incremental cost per additional QALY gained was $\$ 3,709$.
\end{abstract}

Conclusions: PegIFN therapy is safe in HBeAg-positive CHB patients with mild $\mathrm{AE}$, as it results in a higher HBsAg loss rate and longer QALYs than NA therapy.

Keywords: Chronic hepatitis B; Acute exacerbation; Peginterferon $\alpha-2 \mathrm{a}$; Entecavir; Telbivudine

\section{Introduction}

Hepatitis B virus (HBV) infection is the 15th leading cause

Manuscript accepted for publication January 18, 2017

aLiver Research Center, The First Affiliated Hospital of Fujian Medical University, Fuzhou 350005, China

${ }^{b}$ Clinical Liver Center of the 180th Hospital of People's Liberation Army, Quanzhou 362100, China

${ }^{\mathrm{c} C a r d i o l o g y}$ Department, The First Affiliated Hospital of Fujian Medical University, Fuzhou 350005, China

${ }^{\mathrm{d}}$ Corresponding Author: Yueyong Zhu, Liver Research Center, The First Affiliated Hospital of Fujian Medical University, Fuzhou 350005, China.

Email: zhuyueyong@fjmu.edu.cn

doi: https://doi.org/10.14740/gr754w of death worldwide [1]. The nature of HBV infection depends on the state of interactions among the virus, hepatocytes, and the host's immune system. A vigorous immune response may lead to acute exacerbation (AE) [2], which is characterized as alanine aminotransferase (ALT) levels elevated to more than 10 times the upper limit of normal (ULN) or more than twice the baseline level [3].

The treatment of AE is still controversial. Although higher ALT levels indicate a higher spontaneous hepatitis B envelope antigen $(\mathrm{HBeAg})$ seroconversion rate $[3,4]$, about $41.9 \%$ of AE patients without antiviral therapy will have a hepatitis flare within 2 years [5]. Since the repeated hepatitis flare may lead to fibrosis and cirrhosis, antiviral treatment in $\mathrm{AE}$ patients is necessary.

Oral nucleos(t)ide analogues (NA) are widely used antiviral agents in treatment of AE [6,7]. Results from a metaanalysis suggested that NA provided better antiviral responses but no obvious impact on short-term survival in patients with $\mathrm{AE}$ of CHB [7]. However, virological relapse happens after treatment discontinuation, even when $\mathrm{HBeAg}$ loss has been achieved [8]. Relapse and drug resistance are also major challenges of NA therapy [9-11].

Interferon (IFN) has some advantages over NA in treating $\mathrm{CHB}$, such as finite duration of therapy, higher seroconversion rates, and absence of resistance $[12,13]$. IFN was thought to be contraindicated in CHB with AE due to concerns regarding hepatic decompensation during IFN-based treatment [3]. To date, only three published papers had focused on IFN therapy for AE patients [5, 14, 15]. In those studies, entecavir (ETV) was the most frequently used NA. Telbivudine (LDT) has been considered superior to ETV because it has a higher HBeAg seroconversion rate [16]. However, the efficacy of LDT remains unknown in AE patients.

To address those issues, we conducted a retrospective cohort study to evaluate the safety and efficacy of antiviral therapy, including peginterferon $\alpha-2 \mathrm{a}$ (pegIFN), ETV and LDT, on $\mathrm{HBeAg}$-positive patients with AE. A decision tree analysis incorporated Markov processes was used to compare the cost-effectiveness of pegIFN and ETV therapies in those populations.

\section{Patients and Methods}

\section{Patients}

HBeAg-positive CHB patients with mild AE who were admitted to the Liver Research Center of the First Affiliated Hospital of Fujian Medical University from 2008 to 2014 were retro- 
Table 1. Annual Transition Probabilities by Initial State

\begin{tabular}{|llll}
\hline Annual transitional probabilities & Estimated (\%) & Range & References \\
\hline $\begin{array}{l}\text { From HBsAg clearance to } \\
\text { HCC }\end{array}$ & 0.039 & & {$[19]$} \\
From HBeAg Positive CHB to & & & \\
Spontaneous seroconversion & 7.0 & $2.0-23$ & {$[18]$} \\
Compensated cirrhosis & 2.4 & $2.1-2.6$ & {$[18]$} \\
HCC & 0.8 & $0.5-1.0$ & {$[18]$} \\
Death & 0.6 & $0.2-0.9$ & {$[18]$} \\
From HBeAg seroconversion to & & & \\
HBsAg seroclearance & 0.7 & $0.4-1.2$ & {$[18]$} \\
Compensated cirrhosis & 1.0 & $0.1-6.3$ & {$[18]$} \\
HCC & 0.2 & $0.05-0.9$ & {$[18]$} \\
From compensated cirrhosis to & & & \\
Decompensated cirrhosis & 3.9 & $3.2-4.6$ & {$[18]$} \\
HCC & 5.0 & $3.0-7.0$ & {$[18]$} \\
Death & 5.6 & $3.1-8.0$ & {$[18]$} \\
\hline From decompensated cirrhosis to & & & {$[18]$} \\
\hline HCC & 7.1 & $3.5-10.0$ & {$[18]$} \\
Death & 15 & $9.9-20.0$ & {$[18]$} \\
From HCC to & 54.5 & $20.0-60.0$ & {$[18]$} \\
Death & & & \\
\hline
\end{tabular}

HBsAg: hepatitis B surface antigen; HBeAg: hepatitis B envelope antigen; CHB: chronic hepatitis B; HCC: hepatocellular carcinoma.

spectively studied.

The inclusion criteria were: hepatitis B surface antigen (HBsAg)-positive for at least 6 months; HBeAg-positive; with mild AE before antiviral therapy; naive to IFN or NA therapy.

Mild AE was defined as ALT > $10 \mathrm{ULN}$, total bilirubin $(\mathrm{TBIL})<2$ ULN and prothrombin time/international normalized ratio (PT/INR) within the normal range.

Exclusion criteria were pregnancy; cirrhosis; hepatocellular carcinoma (HCC); co-infection with hepatitis D virus, hepatitis $\mathrm{C}$ virus, hepatitis $\mathrm{E}$ virus or human immunodeficiency virus; comorbidities with alcoholism, autoimmune, and metabolic liver disease; patients with antiviral therapy other than pegIFN, ETV, or LDT.

The study protocol was approved by the Institutional Ethics Committee of the First Affiliated Hospital of Fujian Medical University and was in compliance with the Declaration of Helsinki. Written informed consent for the use of clinical data was obtained from each participant.

\section{Treatment}

All patients received glycyrrhizin treatment during hospitalization as it has been demonstrated to rapidly improve serum transaminases in AE patients [17]. Before treatment, all patients were informed of the advantages and disadvantages of receiving pegIFN, ETV, or LDT. The regimens were decided by the patients. For patients who chose NA, ETV $0.5 \mathrm{mg}$ daily or LDT $600 \mathrm{mg}$ daily were initiated once they decided. For those who chose IFN, pegIFN $180 \mu \mathrm{g}$ weekly was added when the ALT declined below 10 ULN. During this waiting period, liver function and PT were monitored every 5 - 7 days. One patient experienced a hepatitis flare with ALT 20 ULN and TBIL 3 ULN while waiting for pegIFN treatment. ETV was initiated as soon as the flare was noticed. The case was not included in final statistics.

The treatment duration of NA patients was at least 96 weeks, and patients were kept on treatment after data collection ended. The duration of pegIFN was 48 weeks in majority of the patients (four patients with 24 weeks' therapy, one with 36 weeks, 49 with 48 weeks and nine with 72 weeks) and patients were followed up until 96 weeks.

According to the treatment regimens, patients were divided into the pegIFN group and the NA group (including LDT and ETV groups).

\section{Data collection}

The baseline characteristics of patients, including gender, age, pathologic result of liver biopsy, HBV DNA, and serologic markers of $\mathrm{HBV}$, were collected from medical records. Liver functions, HBV DNA levels, semi-quantitation $\mathrm{HBeAg}$ and anti-HBe, quantitative $\mathrm{HBsAg}$, and anti-HBs were tested every 12 weeks. In patients receiving pegIFN therapy, the complete 
blood counts and the thyroid function test were also monitored. Patients lost to follow-up were recorded as non-responders. An intent-to-treat (ITT) analysis was used for data analysis.

\section{Laboratory tests and definition}

HBV DNA was performed using LightCycler 480 Real-Time PCR (Roche Diagnostics, USA). HBV DNA negativity was defined as HBV DNA levels $<500 \mathrm{IU} / \mathrm{mL}$. Serologic markers of HBV were measured using the ARCHITECT platform (Abbott Laboratories, Chicago, IL). HBsAg loss was defined as HBsAg titer $<0.05 \mathrm{IU} / \mathrm{mL}$.

$\mathrm{HBsAg} / \mathrm{HBeAg}$ seroconversion was defined as HBsAg/ $\mathrm{HBeAg}$ loss with concomitant appearance of anti-HBs/antiHBe. ALT normalization was defined as ALT levels < 40 IU/L. Virological breakthrough was defined as increase in serum HBV DNA by $>1 \log 10$ (10-fold) above the lowest value after achieving virological response during continued treatment.

\section{Statistical analysis}

Continuous variables were expressed as mean with standard deviation (SD) or the median with interquartile range. Percentage was used for categorical variables. Group comparison was performed using Student's $t$-test, Chi-square test, or Fisher's exact test as appropriate. All tests of significance were twosided, and a $P$ value $<0.05$ was considered statistically significant. Data analyses were performed using SPSS 13.0 software for Windows (SPSS Inc., Chicago, IL, USA).

\section{Cost-effectiveness analysis}

A decision tree analysis, which incorporated Markov processes, was used to simulate the economic outcomes for patients with different treatment strategies after 96 weeks. The model was composed of seven mutually exclusive health states: HBsAg clearance, $\mathrm{HBeAg}$ seroconversion, $\mathrm{HBeAg}$ positive $\mathrm{CHB}$, compensated cirrhosis, decompensated cirrhosis, HCC, and death. Since the clinical data of LDT in AE patients were limited, and LDT did not show a better efficacy in the present study, the comparison of economic outcomes was only made between pegIFN and ETV therapy by cost-effectiveness analysis. Because drug resistance was rare in nucleos(t)ide-naive patients with ETV therapy and theoretically zero in IFN, we did not calculate the health state of virological resistance in this model. The initial state of the model was HBeAg-positive CHB.

We conducted a meta-analysis to determine the mean values of the HBsAg clearance rate and $\mathrm{HBeAg}$ seroconversion rate of pegIFN or ETV therapy based on the data from previous reports $[5,14]$ and the results of the current study. The meta-analysis was performed using Stata version 12.0 (Stata Corp, College Station, TX, USA). The annual transition probabilities were achieved from the recent publications [18, 19] (Table 1). Since most AE patients were in their 30 s and 40 s in most studies, we used the probabilities of the 31 - 40 years' age
Table 2. Annual Costs of Follow-Up or Treatment and Health State Utilities (Quality of Life) in Different Stages of Disease

\begin{tabular}{lll}
\hline & $\begin{array}{l}\text { Health state costs, } \\
\text { RMB (Dollar) [18] }\end{array}$ & $\begin{array}{l}\text { Health state } \\
\text { utilities [20] }\end{array}$ \\
\hline HBsAg clearance & $110(\$ 19)$ & 0.99 \\
HBeAg seroconversion & $110(\$ 19)$ & 0.95 \\
HBeAg-positive CHB & $1,162(\$ 170)$ & 0.85 \\
Compensated cirrhosis & $1,514(\$ 222)$ & 0.69 \\
Decompensated cirrhosis & $13,927(\$ 2,040)$ & 0.35 \\
HCC & $38,795(\$ 5,682)$ & 0.38 \\
\hline
\end{tabular}

HBsAg: hepatitis B surface antigen; HBeAg: hepatitis B envelope antigen; CHB: chronic hepatitis B; HCC: hepatocellular carcinoma.

group if the probabilities differed with age.

As the treatment duration was typically 96 weeks for the ETV group and 48 weeks for the pegIFN group in those studies, the initial costs of drugs were calculated as follows: 1-year pegIFN therapy was US\$10,733 (62,140 RMB), and 2-year ETV therapy was US\$4,410 (25,536 RMB). The annual costs of follow-up or treatment in different state of disease were achieved from Toy's research [18] (Table 2). The health state utilities were based on Levy's research [20] (Table 2). Both costs and health outcomes in the model were discounted at 3\% annually to allow for current values. The stage cycle was 40 .

Outcomes from the model included lifetime costs, qualityadjusted life years (QALYs), and incremental cost-effectiveness ratios (ICERs) gained for the pegIFN treatment compared to ETV treatment.

The analysis was performed with TreeAge Pro 2011 software (TreeAge Software, Williamstown, MA).

\section{Results}

\section{Baseline characteristics of patients}

From 2008 to 2014, $247 \mathrm{HBeAg}$-positive CHB patients with mild AE were selected in this cohort. Seventy-three patients with lamivudine, adefovir dipivoxil, natural INF- $\alpha$, or pegIFN$\alpha-2 b$ therapies and 32 without antiviral therapy were ruled out, as well as one patient with a hepatitis flare before treatment (Fig. 1). A total of 63 patients with pegIFN, 38 with ETV, and 40 with LDT were eligible for analysis.

The entire patient population consisted of 116 males and 25 females, with median ages of $28(23-34)$ years. Fifty-nine patients $(41.84 \%)$ had liver biopsy before treatment. The genotypes of those patients were B or C. The clinical characteristics and outcomes of three groups are shown in Tables 3 and 4.

\section{Safety}

The median pre-treatment duration was $13(7-17)$ days in the pegIFN group. No decompensated hepatitis was found in any of the patients receiving pegIFN therapy on the follow-up. 


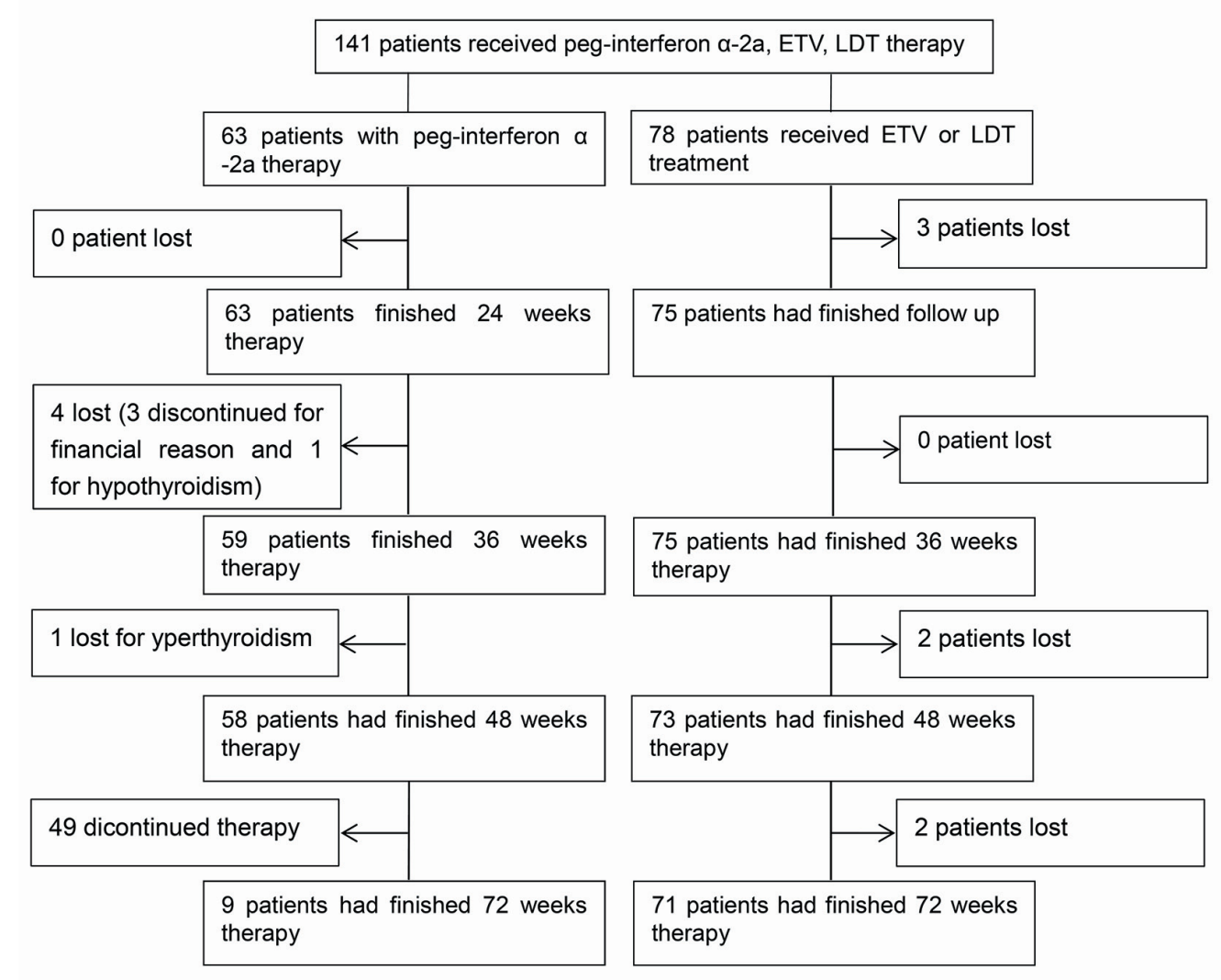

Figure 1. The flowchart of the study design.

Most patients with pegIFN therapy experienced fever in the early period of treatment. The most serious side effect in pegIFN group was hypo- or hyperthyroidism. Two patients had hyperthyroidism on week 36 . One of them discontinued pegIFN therapy because he had achieved HBeAg seroconversion and HBV DNA $<500 \mathrm{IU} / \mathrm{mL}$ on week 36 . The other patient with mild hyperthyroidism received combination therapy with anti-thyroid drugs for another 12 weeks. PegIFN was withdrawn on week 48. One patient experienced hypothyroidism on week 24 and discontinued pegIFN therapy. Three patients discontinued pegIFN therapy for financial reasons after week 24. These patients were included in the final ITT analysis.

No patients in the LDT group experienced myositis or other severe side effects. Two patients in the LDT group experienced virological breakthroughs and switched to ETV combined adefovir dipivoxil therapy. No patients in the ETV group had side effects or virological breakthrough. Seven patients in NA group lost follow-up because they were transferred to local hospital.

\section{Efficacy}

pegIFN vs. $N A$

Patients undergoing NA therapy presented higher ALT normalization and HBV DNA negativity rates than pegIFN therapy on most time points. There was no difference in HBeAg sero- conversion between the two groups (30/63 (47.62\%) vs. $30 / 78$ $(38.46 \%), \mathrm{P}=0.274$ on week 96). However, the HBsAg loss rate was significantly higher on week 72 and week 96 in the pegIFN group when compared with the NA group (7/63 (11.11\%) vs. $1 / 78(1.28 \%), \mathrm{P}=0.022$ on week 72 and $9 / 63(14.29 \%)$ vs. $1 / 78$ (1.28\%), $\mathrm{P}=0.005$ on week 96) (Table 3 and Fig. 2).

None of the patients in the NA group experienced HBsAg seroconversion at the end of follow-up, while three patients in the IFN group had HBsAg seroconversion on week 96.

ETV vs. LDT

Patients in the LDT group had higher ALT normalization rates on weeks 24 and 36. The difference in ALT normalization rates was not significant after week 48 . There was no significant difference in the HBV DNA negativity rate, HBeAg seroconversion, or HBsAg loss/seroconversion rate at any time point between the ETV group and the LDT group. Only one patient in the ETV group achieved HBsAg loss after week 24 (Table 4).

\section{Cost-effectiveness analysis of pegIFN and ETV therapy on $A E$ patients}

From the data of previous reports $[5,14]$ and the results of present study, the calculated HBsAg clearance rate was $16.4 \%$ 
Table 3. The Baseline Characteristics and Outcomes of Acute Exacerbation Patients With Peginterferon a-2a or Nucleos(t)ide Analogues

\begin{tabular}{|c|c|c|c|}
\hline & Peginterferon $\alpha$-2a $(n=63)$ & Nucleos(t)ide analogues $(n=78)$ & Pvalue \\
\hline \multicolumn{4}{|l|}{ Baseline } \\
\hline Male sex, n (\%) & $49 / 63(77.78 \%)$ & $67 / 78(85.9 \%)$ & 0.209 \\
\hline \multicolumn{4}{|l|}{ Biopsy } \\
\hline $\mathrm{G}^{*}$ & $3(2-3)$ & $3(2-3)$ & 0.446 \\
\hline $\mathrm{S}^{*}$ & $3(2-3)$ & $3(2-3)$ & 0.887 \\
\hline Highest & $703(506-1,028)$ & $757(542-1,185)$ & 0.348 \\
\hline Begin pegIFN & $311(280-357)$ & & \\
\hline \multicolumn{4}{|l|}{$\operatorname{TBIL}(\mu \mathrm{mol} / \mathrm{L})$} \\
\hline Highest & $17.62 \pm 5.44$ & $19.10 \pm 5.92$ & 0.154 \\
\hline Begin pegIFN & $13.82 \pm 4.56$ & $15.35 \pm 5.68$ & 0.110 \\
\hline $\mathrm{PT}(\mathrm{s})^{*}$ & $11.80(11.40-12.50)$ & $11.70(11.40-12.43)$ & 0.685 \\
\hline \multicolumn{4}{|l|}{ Results } \\
\hline \multicolumn{4}{|l|}{ ALT normalization, $\mathrm{n} /$ total (\%) } \\
\hline Week 12 & $19 / 63(30.16 \%)$ & $44 / 78(56.41 \%)$ & 0.002 \\
\hline Week 24 & $25 / 63(39.68 \%)$ & $60 / 78(76.92 \%)$ & 0.000 \\
\hline Week 36 & $34 / 63(53.97 \%)$ & $69 / 78(88.46 \%)$ & 0.004 \\
\hline Week 48 & $39 / 63(61.90 \%)$ & $69 / 78(88.46 \%)$ & $<0.001$ \\
\hline Week 72 & $45 / 63(71.43 \%)$ & $69 / 78(88.46 \%)$ & 0.011 \\
\hline Week 96 & $44 / 63(69.84 \%)$ & $68 / 78(87.18 \%)$ & 0.011 \\
\hline \multicolumn{4}{|l|}{ HBV DNA negative, n/total (\%) } \\
\hline Week 12 & $14 / 63(22.22 \%)$ & $26 / 78(33.33 \%)$ & 0.146 \\
\hline Week 24 & $27 / 63(42.86 \%)$ & $47 / 78(60.26 \%)$ & 0.040 \\
\hline Week 36 & $36 / 63(57.14 \%)$ & $55 / 78(70.51 \%)$ & 0.099 \\
\hline Week 72 & $29 / 63(46.03 \%)$ & $27 / 78(34.62 \%)$ & 0.168 \\
\hline Week 96 & $30 / 63(47.62 \%)$ & $30 / 78(38.46 \%)$ & 0.274 \\
\hline \multicolumn{4}{|l|}{ HBsAg loss, n/total (\%) } \\
\hline Week 12 & $0 / 63(0.00 \%)$ & $0 / 78(0.00 \%)$ & \\
\hline Week 24 & $0 / 63(0.00 \%)$ & $1 / 78(1.28 \%)$ & 1.000 \\
\hline Week 36 & $1 / 63(1.59 \%)$ & $1 / 78(1.28 \%)$ & 1.000 \\
\hline Week 48 & $5 / 63(7.94 \%)$ & $1 / 78(1.28 \%)$ & 0.089 \\
\hline Week 72 & $7 / 63(11.11 \%)$ & $1 / 78(1.28 \%)$ & 0.022 \\
\hline Week 96 & $9 / 63(14.29 \%)$ & $1 / 78(1.28 \%)$ & 0.005 \\
\hline \multicolumn{4}{|l|}{ HBsAg seroconversion, $\mathrm{n} /$ total (\%) } \\
\hline Week 12 & $0 / 63(0.00 \%)$ & None & \\
\hline Week 24 & $0 / 63(0.00 \%)$ & & \\
\hline Week 36 & $1 / 63(1.59 \%)$ & & 0.447 \\
\hline Week 48 & $2 / 63(3.17 \%)$ & & 0.198 \\
\hline Week 72 & $3 / 63(4.76 \%)$ & & 0.087 \\
\hline Week 96 & $3 / 63(4.76 \%)$ & & 0.087 \\
\hline
\end{tabular}

HBsAg: hepatitis B surface antigen; HBeAg: hepatitis B envelope antigen; ALT: alanine aminotransferase. *Expressed as median (interquartile range). 
Table 4. The Baseline Characteristics and Outcomes of Acute Exacerbation Patients With ETV or LDT Treatment

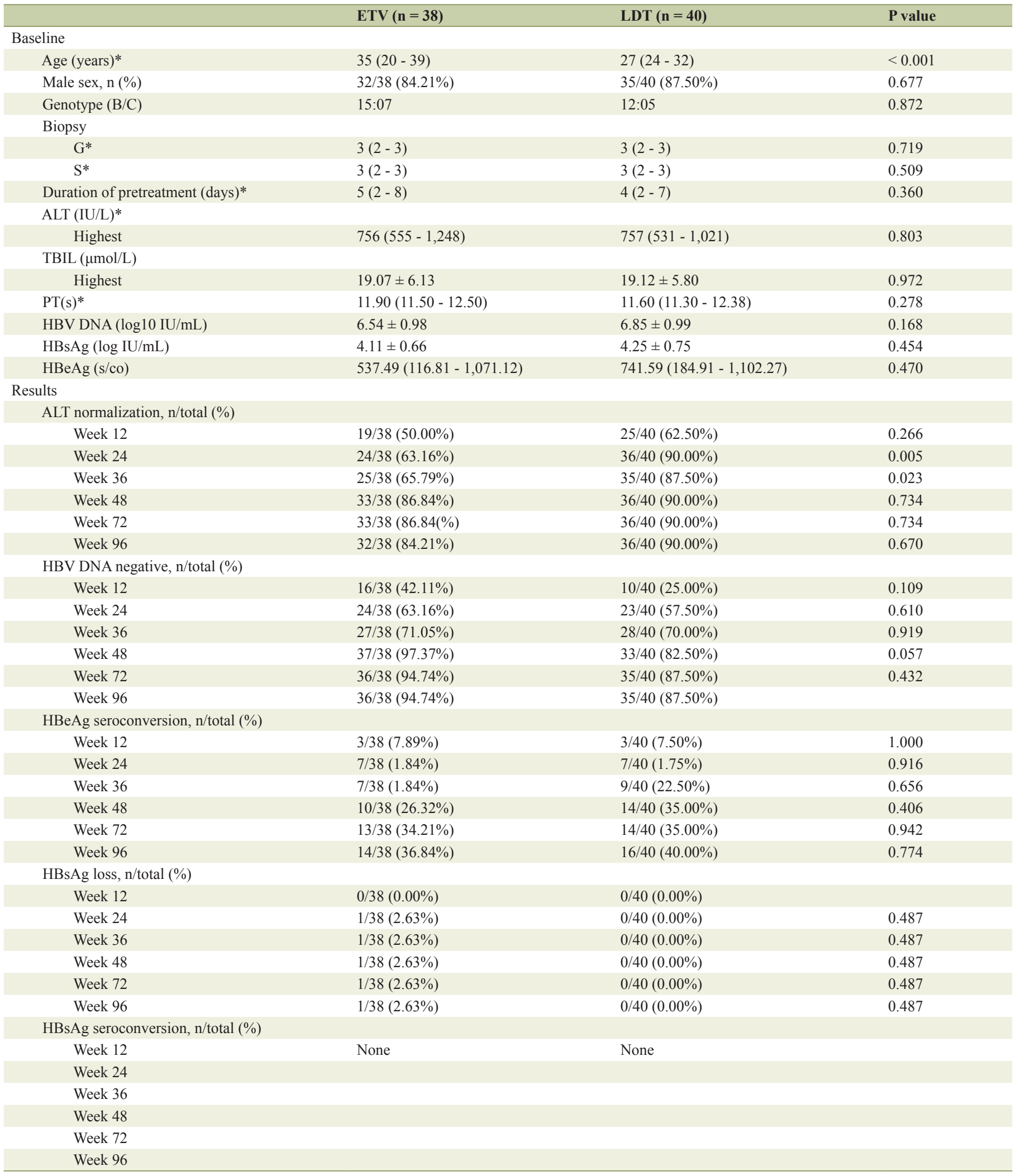

HBsAg: hepatitis B surface antigen; HBeAg: hepatitis B envelope antigen; ALT: alanine aminotransferase. *Expressed as median (interquartile range). 

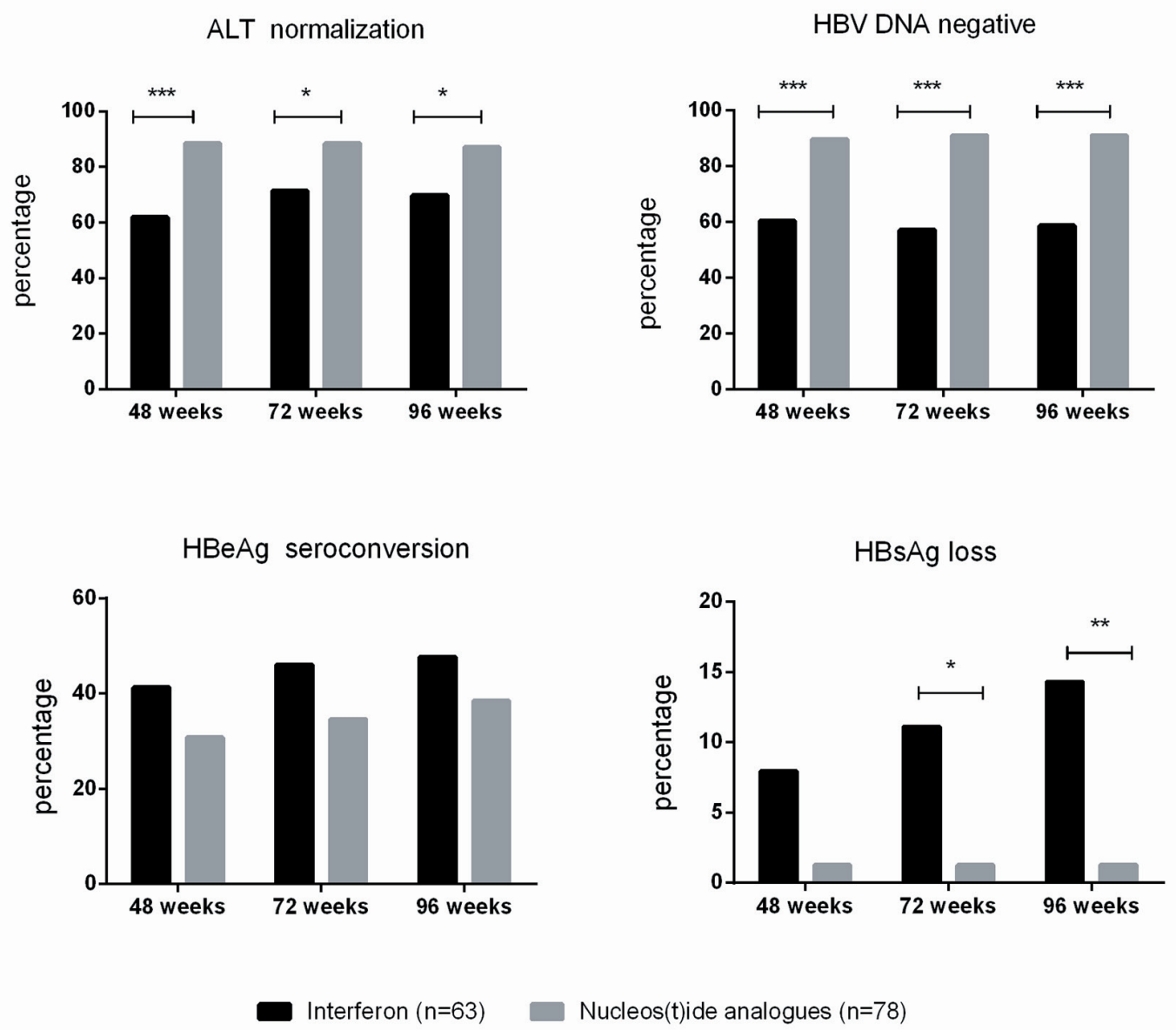

Figure 2. The efficacy of pegIFN and NA treatments in HBeAg-positive $\mathrm{CHB}$ with mild AE. ${ }^{*} \mathrm{P}<0.05$, ${ }^{* *} \mathrm{P}<0.01$, ${ }^{* *} \mathrm{P}<0.001$.

$(9.1-23.7 \%)$ in the pegIFN group and $2.6 \%(2.5-7.7 \%)$ in ETV group. The HBeAg seroconversion rate was $55.0 \%$ (38.8$71.3 \%)$ in the pegIFN group and $40.2 \%(29.2-51.1 \%)$ in the ETV group. In the base-case analysis, pegIFN treatment was the most cost-effective strategy for $\mathrm{HBeAg}$-positive $\mathrm{CHB}$ patients with AE. One year of pegIFN therapy would cost US\$12,837, and result in 18.56 QALYs per patient, compared to US\$7,215 and 17.04 QALYs for 2 years of ETV therapy. The incremental cost per additional QALY gained from pegIFN compared to ETV was \$3,709.

\section{Discussion}

In this study, $\mathrm{HBeAg}$-positive AE patients in the pegIFN group achieved higher HBsAg loss rates when compared with the NA therapy group (HBsAg loss rates: $14.29 \%$ vs. $1.28 \%, \mathrm{P}=$ $0.005)$, which was consistent with the previous reports $(31.8 \%$ and $15.4 \%$ for pegIFN therapy and $0 \%$ for ETV therapy) [5, 14].

The optimal endpoint of CHB is HBsAg seroconversion plus HBV DNA suppression, as HBsAg clearance can greatly decrease the risks of cirrhosis and HCC [21, 22]. However, in patients receiving NA therapy, virological relapse occurs after treatment discontinuation, even when $\mathrm{HBeAg}$ loss has been achieved [8]. PegIFN is better than NA for AE patients when the higher HBsAg loss rate is considered. Although the initial cost of pegIFN was higher than ETV, pegIFN therapy still gained a longer QALY and higher ICER than ETV therapy. Therefore, in $\mathrm{HBeAg}$-positive $\mathrm{CHB}$ patients with $\mathrm{AE}$, if there was no strong contraindication except an elevated ALT level, pegIFN offered another treatment choice.

The major concern about pegIFN therapy in AE patients was the safety during the treatment. In this cohort, for patients who chose pegIFN, the ALT, bilirubin and PT levels were carefully monitored during the waiting period. PegIFN was initiated until the ALT was declined under 10 ULN. Only one patient had TBIL > 2 ULN, and without decompensated liver function after the initiation of ETV therapy, during the waiting period. Actually, most patients with exacerbations have a good prognosis, and only $8 \%$ of them develop hepatic decompensation [23]. The mortality due to AE is uncommon, occurring in only $0.7 \%$ of patients. We carefully selected non-cirrhotic patients with bilirubin levels $<2 \mathrm{ULN}$, and no severe hepatitis or liver failure was documented during treatment. All side effects were typical for IFN therapy. Taken together, pegIFN therapy was safe and tolerable for patients with AE.

LDT is considered to be superior to ETV because of a 
higher $\mathrm{HBeAg}$ loss and seroconversion rate in the general population [16]. In this study, there was no difference between these two groups in either HBV DNA suppression or $\mathrm{HBeAg}$ seroconversion, while two patients with LDT therapy experienced virological breakthrough on week 96. However, because of the small sample size of this study, this result requires further study.

There were several limitations of this study. At first, although patients were prospectively followed, the retrospective nature of this study still compromises the reliability of this study, although we tried to fill the gap by using ITT analysis. Second, it was a single center study. However, the sample size in this cohort was larger than the other studies focusing on pegIFN therapy in the HBeAg-positive AE population. A welldesigned, adequate study is required in the future to confirm the findings in this study.

\section{Conclusion}

In conclusion, pegIFN therapy is safe and more effective than NA therapy in $\mathrm{HBeAg}$-positive $\mathrm{CHB}$ patients with mild $\mathrm{AE}$ as it provides a higher HBsAg loss rate and longer QALYs. LDT does not lead to a higher serological response when compared with ETV therapy.

\section{Financial Disclosures}

$\mathrm{Su}$ Lin has received financial support from the Pilot Project of the Fujian Science and Technology Department (2016Y0040).

\section{Author Contributions}

Su Lin, Yueyong Zhu, and Zhiyuan Weng designed the research study and wrote the paper. Mingfang Wang, Qiaoxia Ye, and Yinlian Wu collected and analyzed the data.

\section{Abbreviations}

HBV: hepatitis B virus; AE: acute exacerbation; ALT: alanine aminotransferase; ULN: upper limit of normal; CHB: chronic $\mathrm{HBV}$; IFN: interferon; NA: nucleos(t)ide analogues; pegIFN: peginterferon $\alpha$-2a; ETV: entecavir; LDT: telbivudine; TBIL: total bilirubin; HCC: hepatocellular carcinoma; QALYs: quality-adjusted life years; ICERs: incremental cost-effectiveness ratios

\section{References}

1. Lozano R, Naghavi M, Foreman K, Lim S, Shibuya K, Aboyans V, Abraham J, et al. Global and regional mortality from 235 causes of death for 20 age groups in 1990 and 2010: a systematic analysis for the Global Burden of Disease Study 2010. Lancet. 2012;380(9859):2095-2128.
2. Yang Q, Shi Y, Yang Y, Lou G, Chen Z. The sterile inflammation in the exacerbation of HBV-associated liver injury. Mediators Inflamm. 2015;2015:508681.

3. Lok AS, McMahon BJ. Chronic hepatitis B: update 2009. Hepatology. 2009;50(3):661-662.

4. Yuen MF, Yuan HJ, Hui CK, Wong DK, Wong WM, Chan $\mathrm{AO}$, Wong BC, et al. A large population study of spontaneous $\mathrm{HBeAg}$ seroconversion and acute exacerbation of chronic hepatitis B infection: implications for antiviral therapy. Gut. 2003;52(3):416-419.

5. Cai Q, Chen F, Shao X, Zhang X, Zhao Z, Gao Z. Treatment of pegylated interferon-alpha2a in chronic hepatitis B patients demonstrating a spontaneous decline in HBV DNA after acute exacerbation. Antivir Ther. 2015;20(2):217-224.

6. Hung $\mathrm{CH}, \mathrm{Hu} \mathrm{TH}$, Lu SN, Lee CM, Chen $\mathrm{CH}$, Kee KM, Wang JH, et al. Tenofovir versus entecavir in treatment of chronic hepatitis B virus with severe acute exacerbation. Antimicrob Agents Chemother. 2015;59(6):3168-3173.

7. $\mathrm{Yu} \mathrm{W,} \mathrm{Zhao} \mathrm{C,} \mathrm{Shen} \mathrm{C,} \mathrm{Wang} \mathrm{Y,} \mathrm{Lu} \mathrm{H,} \mathrm{Fan} \mathrm{J.} \mathrm{The} \mathrm{ef-}$ ficacy and safety of Nucleos(t)ide analogues in patients with spontaneous acute exacerbation of chronic hepatitis B: a systematic review and meta-analysis. PLoS One. 2013;8(6):e65952.

8. Perez-Cameo C, Pons M, Esteban R. New therapeutic perspectives in HBV: when to stop NAs. Liver Int. 2014;34(Suppl 1):146-153.

9. Wong VW, Chan HL. Severe acute exacerbation of chronic hepatitis B: a unique presentation of a common disease. J Gastroenterol Hepatol. 2009;24(7):1179-1186.

10. Devi U, Locarnini S. Hepatitis B antivirals and resistance. Curr Opin Virol. 2013;3(5):495-500.

11. Lee IC, Sun CK, Su CW, Wang YJ, Chang HC, Huang $\mathrm{HC}$, Lee KC, et al. Durability of Nucleos(t)ide Analogues Treatment in Patients With Chronic Hepatitis B. Medicine (Baltimore). 2015;94(32):e1341.

12. EASL clinical practice guidelines: Management of chronic hepatitis B virus infection. J Hepatol. 2012;57(1):167185.

13. Zhou P, Yang F, Wang J, Mao R, Qi X, Huang Y, Zhang $\mathrm{J}$. Improved Efficacy of a pegylated interferon-alpha-2a stepwise optimization treatment strategy in the treatment of hepatitis B e antigen-positive chronic hepatitis B patients. Medicine (Baltimore). 2015;94(17):e730.

14. Chen CC, Wang PC, Chang HW, Chen CF. Safety and efficacy of two-step peginterferon alpha-2a treatment in patients of chronic hepatitis B with acute exacerbation. J Viral Hepat. 2012;19(3):161-172.

15. Okuse C, Yotsuyanagi H, Yamada N, Okamoto M, Ikeda H, Kobayashi M, Fukuda Y, et al. Effect of nucleoside analog-interferon sequential therapy on patients with acute exacerbation of chronic hepatitis B. Hepatol Res. 2010;40(5):461-469.

16. Liu H, Wang X, Wan G, Yang Z, Zeng H. Telbivudine versus entecavir for nucleos(t)ide-naive HBeAg-positive chronic hepatitis B: a meta-analysis. Am J Med Sci. 2014;347(2):131-138.

17. Lin CC, Wang PH. Intravenous glycyrrhizin improved serum transaminases rapidly in a chronic hepatitis B 
patient with acute exacerbation. J Formos Med Assoc. 2015;114(2):188-189.

18. Toy M, Salomon JA, Jiang H, Gui H, Wang H, Wang J, Richardus JH, et al. Population health impact and cost-effectiveness of monitoring inactive chronic hepatitis B and treating eligible patients in Shanghai, China. Hepatology. 2014;60(1):46-55.

19. Locarnini S, Hatzakis A, Chen DS, Lok A. Strategies to control hepatitis B: Public policy, epidemiology, vaccine and drugs. J Hepatol. 2015;62(1 Suppl):S76-86.

20. Levy AR, Kowdley KV, Iloeje U, Tafesse E, Mukherjee J, Gish R, Bzowej N, et al. The impact of chronic hepatitis B on quality of life: a multinational study of utili- ties from infected and uninfected persons. Value Health. 2008;11(3):527-538.

21. Lauret E, Gonzalez-Dieguez ML, Rodriguez M, Gonzalez M, Melon S, Rodrigo L. Long-term outcome in Caucasian patients with chronic hepatitis B virus infection after HBsAg seroclearance. Liver Int. 2015;35(1):140-147.

22. Kim GA, Lee HC, Kim MJ, Ha Y, Park EJ, An J, Lee D, et al. Incidence of hepatocellular carcinoma after HBsAg seroclearance in chronic hepatitis B patients: a need for surveillance. J Hepatol. 2015;62(5):1092-1099.

23. Tsai WL, Sun WC, Cheng JS. Chronic Hepatitis B with Spontaneous Severe Acute Exacerbation. Int J Mol Sci. 2015;16(12):28126-28145. 